\title{
An Aberrant Origin of the Right Hepatic Artery: A Rare Anatomic Variation. And Its Clinical Application
}

\author{
Sanaa Al-Shaarawy, Essam Eldin Abdelhady Salama \\ Department of Anatomy, College of Medicine, King Saud University, Riyadh, KSA \\ Email: salsharawi@ksu.edu.sa, esalama@ksu.edu.sa, essamco58@gmail.com
}

How to cite this paper: Al-Shaarawy, S. and Salama, E.E.A. (2022) An Aberrant Origin of the Right Hepatic Artery: A Rare Anatomic Variation. And Its Clinical Application. Forensic Medicine and Anatomy Research, 10, 7-12.

https://doi.org/10.4236/fmar.2022.101002

Received: November 13, 2021

Accepted: December 12, 2021

Published: December 15, 2021

Copyright $\odot 2022$ by author(s) and Scientific Research Publishing Inc. This work is licensed under the Creative Commons Attribution International License (CC BY 4.0).

http://creativecommons.org/licenses/by/4.0/

\begin{abstract}
The right hepatic artery is an important arterial supply to right lobe of the liver. And the knowledge of the normal anatomy and anatomical variations of the right hepatic artery is essential to perfume, and will minimize morbidity, and also help to decrease the number of complications of hepatobiliary surgery. This study was conducted on eleven human cadavers, which were obtained from the routine autopsies at the dissection room of the Anatomy Department. During dissection of the eleven cadaveric livers, we found a case with an exceptional anatomic variation; a replaced right hepatic artery (RRHA) coming off the superior mesenteric artery (SMA), directly to the hepatic right lobe passing through the Calot's triangle, crossing behind the common hepatic duct (CHD). Our objective is to draw much attention to this particularly anatomic variation of the origin of the RRHA as well as its clinical importance in order to ensure that no damage will be made during gastrointestinal and hepatobiliary surgery.
\end{abstract}

\section{Keywords}

Right Hepatic Artery, Anatomic Variation, Superior Mesenteric Artery, Calot's Triangle, Hepatobiliary Surgery

\section{Introduction}

The anatomy of the hepatic artery is of great importance in hepatobiliary surgery, especially in cholecystectomy and liver transplantation. The celiac trunk (CT) is the first ventral branch of the abdominal aorta arising at T12/L1 vertebral body level; it passes forward dividing into left gastric artery, common hepatic artery (CHA) and splenic artery, which is the classic distribution pattern 
[1] [2]. The CHA is recently defined as an arterial trunk containing at least one segmental hepatic artery and the gastroduodenal artery, irrespective of its origin and course; this definition facilitates the study of different (CHA) anomalies [3]. The superior mesenteric artery (SMA) is the second ventral branch of the abdominal aorta arising below the level of the celiac trunk; it gives inferior pancreaticoduodenal, middle colic, right colic, ileocolic, jejunal and ileal branches [4]. The hepatic artery (Proper hepatic artery-PHA) is the branch of the celiac trunk passing forwards and to the right to the upper surface of the first part of the duodenum; it lies in front of portal vein and to the left side of the bile duct; it curves upwards and ascends between the two layers of the lesser omentum to enter the porta hepatis where it divides into two terminal branches, the right hepatic artery (RHA) and the left hepatic artery (LHA). The RHA usually arises from the proper hepatic artery (PHA) which is a continuation of common hepatic artery (CHA), usually to the left of the common hepatic duct (CHD). The RHA runs upward, crossing behind the (CHD) to enter hepatocystic triangle; it gives off the cystic artery (CA) and then turns upward to enter the right lobe of the liver, dividing into an anterior and a posterior branches for segmental supply of the liver and the gall bladder [1] [2]. The knowledge of the anatomical variants in hepatic vascular structures is of great importance in general surgery, laparoscopic surgery, radiological procedures, and the treatment of penetrating injuries involving the peri-hepatic area [5]. When the (RHA) does not arise from the (PHA) or (CHA), its origin is shifted to the aorta or any of the arteries whose normal course is towards right side of the aorta like superior mesenteric artery (SMA), gastroduodenal artery, right gastric artery, or celiac trunk [6] [7]. Michel [8] described the hepatic arterial anatomy and its variations using the results of 200 cadaveric dissections and identified 10 types of hepatic arterial anatomy; type III is the origin of the RRHA from the SMA. The Michel's classification was modified by Hiatt who distinguishes six categories; the (RRHA) arising from the (SMA)-Hiatt type III, [9] [10]. The (RRHA) comprises $11 \%-21 \%$ of cases and replaced (LHA) 3.8\% - 10\% of cases, while accessory RHA and LHA have a frequency of $0.8 \%-8 \%$ according to a recent review [11]. Embryologically, the celiac trunk derives from six pairs of ventral splanchnic vessels (subphrenic, upper, middle, lower ventricular and upper and lower intestinal), and during the fetal development, these pairs disappear. However, the persistence of longitudinal channels between primitive vessels may lead to vascular anomalies or variations [12].

\section{Material and Methods}

Anatomical variation in the origin of the right hepatic artery was looked for in eleven human cadavers during routine dissection on the abdominal region carried out in the Dissection Room of the Anatomy Department, College of Medicine, King Saud University. Variation in the origin and course of the RRHA as well as its relations with the hepatic ducts was recorded and appropriate photograph was taken. 


\section{Results}

The origin of the right hepatic artery (RHA) from the proper hepatic artery (PHA) represents the majority of cases (10 cadavers) (Figure 1). The replaced right hepatic artery (RRHA) was seen arose from (SMA), seen entered the Calot's triangle, and crossed posteriorly to the common hepatic duct (CHD) (Figure 2).

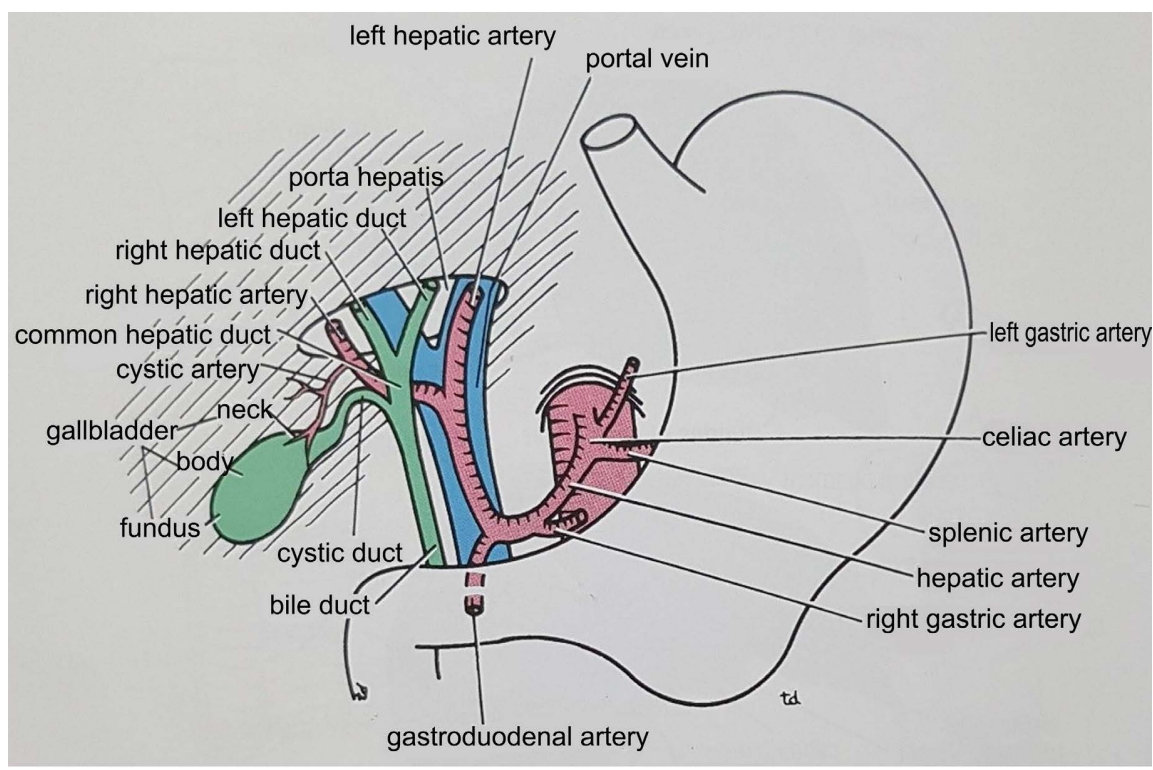

Figure 1. Origen of the RHA from PHA as shown with structures entering and leaving the porta hepatis (Snell [13]).

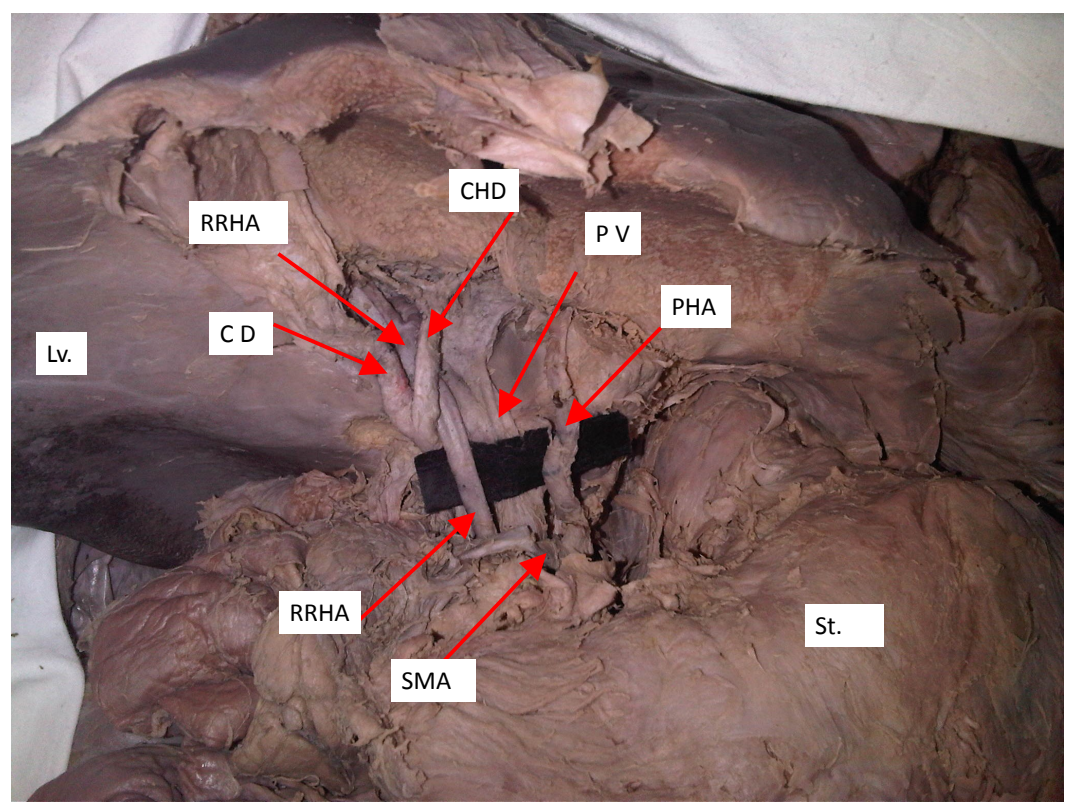

Figure 2. (CA) Cystic artery, (CD) cystic duct, (CHD) common hepatic duct, (PV) portal vein, (PHA) proper hepatic artery, (RHA) right hepatic artery, (SMA) superior mesenteric artery, (St) stomach, (Lv) liver. The course of (RRHA) arising from (SMA), is clear, passing through Calot's triangle, crossing behind the (CHD). 


\section{Discussion}

Our resulting study demonstrated that the (RRHA) originated from the (SMA), which is in agreement with type III Michel's classification [8], and the later modification as Hiatt type III [9] [10]. Also, our result comprises about $9 \%$ of the dissected cases which is in agreement with the results documented by Armaki et al. [4], which is ranged between $5 \%-25 \%$ of reported cases. The posterior relation of the RRHA to CHD which demonstrated in this work is in agreement with literatures approved by Johnston and Anson [14] and Bhargava et al. [15]. These anatomic variants are explained developmentally; that during embryonic development, the aorta gives off ventral segments, four of which become the celiac, splenic, common hepatic, and superior mesenteric arteries, a longitudinal ventral artery anastomoses these segments. So, the replaced right hepatic artery (RRHA) originates from the persistence of the longitudinal ventral arterial segment connected to the superior mesenteric artery (SMA), so that, it can be attributed to the abnormal persistence or regression of an embryonic artery [12] [16]. Chiang et al. [17] described the anatomical view of the aberrations that are proved by the angiographic studies of hepatic arteries This variant is of no clinical meaning unless the (SMA) becomes compressed, or occluded, which is a common clinical problem, and if the patient possesses a (RRHA), not only the gut but also the liver will become necrotic. Patients with (SMA) embolism often present clinically with atrial fibrillation and persistent abdominal pain, and if the collateral circulation fails, (SMA) occlusion results in life threatening intestinal necrosis [18]. Studies suggest that a (RRHA) is a welcome discovery in right liver living donors, a detrimental consequence of living donor transplantation is a shorter and thinner hepatic artery graft, since dissections of the right hepatic artery in living donors must be limited to the right side of the common bile duct to prevent its devascularization [4]. The RHA variations and anomalies are not just anatomical study concerns but, it plays an important role in surgical bracts, the (RRHA) may be mistaken for (CA), and is liable to get ligated [15]. The (RRHA), as it crosses the bile ducts near the junction of the (CD), is liable to injury during cholecystectomy [19]. An aberrant RHA arising from the (SMA) or the aorta may run behind the portal vein [2]. In pancreatectomy and in operations on the duodenum, an aberrant RHA may be ligated compromising the blood supply to the right lobe. The accessory RHA may be injured during resection of the pancreatic head because the artery lies in close proximity to the portal vein [1]. Due to the variant course, the RHA comes in close proximity to (CD) and the gall bladder, this results in formation of short (CA); thus, RHA may be mistaken for (CA) and inadvertently ligated during surgical procedures like cholecystectomy and liver transplantation [20] [21]. The presence of (RRHA) can be life-saving in patients with bile duct cancer because it is further away from the bile duct and tends to be spared from the cancer, making excision of the tumor feasible [1]. The danger in case of injury to a (RRHA), is manifested on the effect on the liver, specifically the bile duct vascularity with the concomi- 
tant risk of a leak in the Bili enteric anastomosis, this is because the RHA is the chief source of blood supply to the bile duct. However, the replaced (CHA) arises from the SMA by a common trunk referred to as the hepatomesenteric trunk; accidental ligation of this vessel can lead to ischemia of the Bili enteric anastomosis [22].

\section{Conclusion}

Knowledge of the anatomical variations regarding the origin, course, and destination of the hepatic vasculatures is an important factor to obtain fair results of the hepatobiliary surgeries and to overcome the resultant complications.

\section{Acknowledgements}

Our acknowledgment is to Dr. Sahar Idres for her faithful help for this study.

\section{Conflicts of Interest}

The authors declare no conflicts of interest regarding the publication of this paper.

\section{References}

[1] Standring, S. (2008) Gray's Anatomy: The Anatomical Basis of Clinical Practice. 40th Edition, Churchill Livingstone, New York.

[2] Hollinshead, W.H. (1971) Anatomy for Surgeons: The Thorax Abdomen and Pelvis. 2nd Edition, Harper and Row, New York.

[3] Xu, X. and Zheng, S. (2006) Variations and Reconstruction of the Hepatic Artery in Liver Transplantation. Hepatobiliary \& Pancreatic Diseases International, 15, 170-172.

[4] Aramaki, O., Sugawara, Y., Kokudo, N., Takayama, T. and Makuuchi, M. (2006) Branch Patch Reconstruction in Living Donor Liver Transplantation: Arterialization of Grafts with Replaced Type Arteries. Transplantation, 82, 1541-1543. https://doi.org/10.1097/01.tp.0000236102.36326.a6

[5] Suzuki, T., Nakayasu, A., Kawabe, K., Takeda, H. and Honjo, I. (1971) Surgical Significance of Anatomic Variations of the Hepatic Artery. American Journal of Surgery, 122, 505-512. https://doi.org/10.1016/0002-9610(71)90476-4

[6] Jones, R.M. and Hardy, K.J. (2001) The Hepatic Artery; A Reminder of Surgical Anatomy. Journal of Royal College of Surgeons of Edinburgh, 46, 168-170. https://doi.org/10.1016/0002-9610(71)90476-4

[7] Bhardwaj, N. (2010) Anomalous Origins of Hepatic Artery and Its Significance for Hepatobiliary Surgery. Journal of the Anatomical Society of India, 59, 173-176. https://doi.org/10.1016/S0003-2778(10)80020-6

[8] Michels, N.A. (1966) Newer Anatomy of the Liver and Its Variant Blood Supply and Collateral Circulation. American Journal of Surgery, 112, 337-347. https://doi.org/10.1016/0002-9610(66)90201-7

[9] Paraskevas, G.K. and Raikos, A. (2011) Multiple Aberrant Coeliac Trunk Ramifications. Singapore Medical Journal, 52, e147-e149.

[10] Hiatt, J.R., Gabbay, J. and Busuttil, R.W. (1994) Surgical Anatomy of the Hepatic Arteries in 1000 Cases. Annals of Surgery, 220, 50-52. 
https://doi.org/10.1097/00000658-199407000-00008

[11] Shukla, P.J., Barreto, S.G., Kulkarni, A., Nagarajan, G. and Fingerhut, A. (2010) Vascular Anomalies Encountered during Pancreatoduodenectomy: Do They Influence Outcomes? Annals of Surgical Oncology, 17, 186-193. https://doi.org/10.1245/s10434-009-0757-1

[12] Kosaka, M., Horiuchi, K., Nishida, K., Taguchi, T., Murakami, T. and Ohtsuka, A. (2002) Hepatopancreatic Arterial Ring: Bilateral Symmetric Typology in Human Celiaco-Mesenteric Arterial System. Acta Medica Okayama, 56, 245-253.

[13] Snell, R.S. (2004) Clinical Anatomy. 7th Edition, Lippincott Williams \& Wilkins, Philadelphia, Baltimore, New York, London, Buenos Aires, Hong Kong, Sydney, Tokyo, Chapter 5, p. 213.

[14] Johnston, E.V. and Anson, B.J. (1952) Variations in the Formation and Vascular Relationships of the Bile Ducts. Surgery, Gynecology \& Obstetrics, 94, 669-686.

[15] Bhargava, G.S., Singh, H., Singh, H.D. and Gupta, R. (2014) Moynihan's Hump of Right Hepatic Artery: A Case Report and Surgical Significance. CIBTech Journal of Surgery, 3, 42-44.

[16] Peschaud, F., El-Hajjam, M., Malafosse, R., Goere, D., Benoist, S., Penna, C. and Nordlinger, B. (2006) A Common Hepatic Artery Passing in Front of the Portal Vein. Surgical and Radiologic Anatomy, 28, 202-205. https://doi.org/10.1007/s00276-005-0063-y

[17] Chiang, K., Chang, P., Lee, S., et al. (2005) Angiographic Evaluation of Hepatic Artery Variations in 405 Cases. Chinese Journal of Radiology, 30, 75-81.

[18] Barakate, M.S., Cappe, I., Curtin, A., Engel, K.D., Li-Kim-Moy, J., Poon, M.S. and Sandeman, M.D. (2002) Management of Acute Superior Mesenteric Artery Occlusion. ANZ Journal of Surgery, 72, 25-29. https://doi.org/10.1046/j.1445-2197.2002.02289.x

[19] Flint, E.R. (1923) Abnormalities of the Right Hepatic, Cystic and Gastro Duodenal Arteries and of Bile Ducts. British Journal of Surgery, 10, 509-519. https://doi.org/10.1002/bjs.1800104011

[20] Devi, J., Mugunthan, N., Phalgunan, V. and Shiva, D.S. (2012) Caterpillar Hump of Right Hepatic a Rtery: Incidence and Surgical Significance. National Journal of Clinical Anatomy, 1, 121-124. https://doi.org/10.4103/2277-4025.298016

[21] Nagral, S. (2005) Anatomy Relevant to Cholecystectomy. Journal of Minimal Access Surgery, 1, 53-58. https://doi.org/10.4103/0972-9941.16527

[22] Noussiosa, G., Dimitrioua, I., Chatzisb, I. and Katsourakisb, A. (2017) The Main Anatomic Variations of the Hepatic Artery and Their Importance in Surgical Practice. Journal of Clinical Medicine Research, 9, 248-252. https://doi.org/10.14740/jocmr2902w 